Bull. Austral. Math. Soc.

$65 \mathrm{~N} 15,35 \mathrm{M} 10$

VOL. 56 (1997) [217-225]

\title{
FINITE ELEMENT METHODS FOR COMPRESSIBLE STOKES EQUATIONS WITH INFLOW BOUNDARY CONDITION
}

\section{JAE RYONG KWEON}

A finite element method for solving the compressible viscous Stokes equation with an inflow boundary condition is presented. The unique existence of the solution of the discrete problem is established, and an error analysis is given. It is shown that the error in pressure is dominated by the one in velocity and an error at the inflow portion of the boundary.

\section{INTRODUCTION}

We study and give an error analysis of numerical methods for the stationary linearised barotropic compressible viscous Navier-Stokes system with an inflow boundary condition, using finite element methods. Such a linearised system is often called a "compressible Stokes system" because it may also be obtained from the (incompressible) Stokes equations and certain modifications. It is well-known that the system is not elliptic in the ADN sense: the momentum equations are elliptic in velocity and the continuity one is hyperbolic in pressure, so that it is neither elliptic nor hyperbolic. Recently in $[4,5]$ we have studied the whole nonlinear steady-state barotropic compressible viscous Navier-Stokes equations with an inflow boundary condition. The reason that the inflow boundary condition is considered is: if one assigns specified nonzero values to the velocity components on the boundary of the region, then the velocity field gives the characteristic directions for the hyperbolic continuity equation, and so values of density must be specified on those portions of the boundary where the specified velocity vector points into the region. Naturally in the linearised problem a boundary condition for pressure is imposed, and zero boundary condition for velocity and zero initial condition for pressure are considered because it was convenient to use as dependant variables the deviation from the boundary values.

This paper consider the following problem (called a compressible Stokes system)

$$
\left\{\begin{array}{l}
-\mu \Delta \mathbf{u}-\nu \nabla \operatorname{div} \mathbf{u}+(\mathbf{U} \cdot \nabla) \mathbf{u}+\nabla p=\mathbf{f} \text { in } \Omega, \\
\operatorname{div} \mathbf{u}+\mathbf{U} \cdot \nabla p=\mathbf{0} \text { in } \Omega \\
\mathbf{u}=0 \quad \text { on } \Gamma \\
p=0 \quad \text { on } \Gamma_{\text {in }} .
\end{array}\right.
$$

Received 21st October, 1996

This work was partially supported by GARC-KOSEF. The author would like to give thanks to Prof. R.B. Kellogg for several useful comments and discussions.

Copyright Clearance Centre, Inc. Serial-fee code: 0004-9729/97 \$A2.00+0.00. 
Here $\Omega$ is an open bounded domain in $R^{2}$ with smooth boundary $\Gamma$, and $\mathbf{u}=[u, v]$ is a velocity vector, $p$ is the pressure, $\mathbf{U}=[U, V]$ is a given smooth vector function of $(x, y)$, and $\mathbf{f}$ is a given function. The numbers $\mu$ and $\nu$ are viscous constants with $\mu>0$ and $\mu>-\nu$. Throughout this paper it is assumed that $U \geqslant C_{0}>0$ for a constant $C_{0}$. The incoming and outgoing portions of the boundary, $\Gamma_{\text {in }}$ and $\Gamma_{\text {out }}$ are defined by

$$
\begin{aligned}
\Gamma_{\text {in }} & =\{(x, y) \in \Gamma: \mathbf{U} \cdot \mathbf{n}<0\}, \\
\Gamma_{\text {out }} & =\{(x, y) \in \Gamma: \mathbf{U} \cdot \mathbf{n} \geqslant 0\},
\end{aligned}
$$

where $\mathbf{n}=\left(n_{1}, n_{2}\right)$ denotes the unit outward pointing normal vector to $\Gamma$.

In [5] we showed that the regularity of the solution of (1) depends on the geometry of the boundary of the domain, not on the regularity of the data because the singularities of the solution at the junction of inflow and outflow portions block further regularity in the solution, and we proved some regularity results for the linear and nonlinear problems. The regularity result for the linear case will be given in Section 2 . In this paper we use this result and develop a finite element method for the system (1) by giving some modifications to the continuity equation. It is shown that the error in pressure is bounded by the one in velocity and an error at the inflow portion of the boundary. An error analysis for the finite element method is given.

To make our analysis simple, we assume throughout this paper that $U=1$ and $V=0$. In fact, if $\Gamma$ has nonzero curvature at the points where $\mathbf{U} \cdot \mathbf{n}$ is zero, Theorem 1 in Section 2 can be obtained under suitable assumptions. Hence any bounded domain in $R^{2}$, with a $C^{2}$ boundary which has nonzero curvature at the points where $\mathbf{U} \cdot \mathbf{n}$ is zero, will be appropriate as a domain of the problem (1). Here a standard example of a domain is "a circle" in $R^{2}$ with centre the origin and radius 1 .

In [3], an error analysis was given, and the error bound does not have optimal order in terms of approximation theory; the presence of the convective term in the continuity equation degrades the error bound, and also the problems in [3] have quite different character, namely, the flows just stay and play in a given domain, but the ones in our problem move in and out. In [3], a unique existence of the discrete solution was established by showing that the subspaces for velocity and pressure satisfy the "inf-sup" condition associated with the (incompressible) Stokes system.

Some advantages for the method to be presented here are that we have a nice error analysis for (1), and that in [2] and [3], the bubble function in the "MINI" element subspaces is necessary in proving stability, while in our problem, it is not. So the resulting finite element matrix order is much smaller than the one in [3]. A disadvantage is that the stiffness matrix resulting from our weak formulation is not sparse in some block of the matrix. Furthermore, it has been observed that an approximation of the 
incoming portion boundary plays a role in resolving the accuracy of the discrete solution.

In Section 2 we propose a finite element method for (1), the "streamline" finite element method. The unique existence of the discrete solution for the finite element formulation is shown and its error analysis is given. It would be interesting to determine if the analysis given here for (1) can be extended to the system (1) with the nonconstant vector field $\mathbf{U}=[U, V]$.

In this paper, the following Sobolev spaces and norms shall be used: $H^{2, q}(\Omega)$ is the Sobolev space of real valued $L^{q}$ functions such that their derivatives belong to $L^{q}(\Omega)$. We write $\|u\|_{0, q}$ and $\|u\|_{2, q}$ for the norms in $L^{q}(\Omega)$ and $H^{2, q}(\Omega)$, and $(u, v),\|u\|_{0}$ for the scalar product and norm in $L^{2}(\Omega) . H_{0}^{1}(\Omega)=\left\{u \in H^{1}(\Omega):\left.u\right|_{\Gamma}=0\right\}$ and $H_{0}^{2, q}(\Omega)=H^{2, q}(\Omega) \cap H_{0}^{1}$ and $|u|_{\infty} \equiv \max \{|u(x)|: x \in \Omega\}, L^{\infty}(\Omega)=\left\{u:|u|_{\infty}<\infty\right\}$. We shall frequently use the following Sobolev imbedding result (see [1]): $H^{1, q}(\Omega) \subset L^{\infty}$ as well as the norm inequalities that correspond to this imbedding.

In our proofs, $C$ denotes a generic constant, depending on certain quantities. We shall make this dependence explicit, for example, writing $C(\Omega)$ if $C$ depends only on $\Omega$ (for example, in the Sobolev inequalities) or $C\left(\Omega, \mathrm{U}, C_{0}\right)$ if $C$ depends on $\Omega, \mathrm{U}$, and $C_{0}$, and so on.

\section{Finite element Method}

We start this section by citing the following result from [5, Theorem 2.1].

THEOREM 1. Let $2<q<3$. Suppose that $U \geqslant C_{0}>0$ for some constant $C_{0}$. Assume that $\mu$ is large enough. Then there is a unique solution $[\mathbf{u}, p] \in H_{0}^{2, q} \times H^{1, q}$ of (1). Also the solution $[\mathbf{u}, p]$ satisfies

$$
\|\mathbf{u}\|_{2, q}+\|p\|_{1, q} \leqslant C\left(\|\mathbf{f}\|_{0, q}+\|g\|_{1, q}\right)
$$

where $C=C\left(\Omega,\|\mathbf{U}\|_{2, q}, C_{0},(\mu+\nu) / \mu, \mathbf{U}\right)$ and $g=0$.

We let $\mathbf{V}=H_{0}^{1}(\Omega) \times H_{0}^{1}(\Omega)$ and $M=L^{2}(\Omega)$, and consider a real-valued function $\delta$ defined by $\delta(y)=-\sqrt{1-y^{2}}(-1 \leqslant y \leqslant 1)$. We define a linear map $T: \mathrm{V} \longrightarrow R$ by

$$
T \mathbf{u}(x, y)=\int_{\delta(y)}^{x} \nabla \cdot \mathbf{u}(s, y) d s .
$$

An easy computation shows that $\|T \mathbf{u}\|_{0} \leqslant\|\nabla \mathbf{u}\|_{0}$. With the observations described in the previous section, the domain $\Omega$ to be considered will be a unit circle with centre at the origin, which can be described in this way: $\Omega=\left\{(x, y) \in R^{2}: x^{2}+y^{2}<1\right\}, \Gamma=$ $\left\{(x, y) \in R^{2}: x^{2}+y^{2}=1\right\}$, and $\Gamma_{\text {in }}=\left\{(\delta(y), y): \delta(y)=-\sqrt{\left(1-y^{2}\right)},-1 \leqslant y \leqslant 1\right\}$. 
Hence using (2) with $U=1$ and $V=0$, the system (1) becomes

$$
\begin{aligned}
-\mu \Delta \mathbf{u}-\nu \nabla \operatorname{div} \mathbf{u}+(\mathbf{U} \cdot \nabla) \mathbf{u}+\nabla p=\mathbf{f} & & \text { in } \Omega, \\
p+T \mathbf{u}=\mathbf{0} & & \text { in } \Omega, \\
\mathbf{u}=0 & & \text { on } \Gamma .
\end{aligned}
$$

Notice that (2) implies $p(\delta(y), y)=0$. In order to formulate the problem (3) in a discrete version, several bilinear forms are considered. We define the bilinear form $a$ by

$$
a(\mathbf{u}, \mathbf{v})=\int_{\Omega}\{\mu \nabla \mathbf{u} \cdot \nabla \mathbf{v}+\nu \operatorname{div} \mathbf{u} \operatorname{div} \mathbf{v}+[(\mathbf{U} \cdot \nabla) \mathbf{u}] \mathbf{v}\} \mathrm{d} \mathbf{x}, \quad \mathbf{u}, \mathbf{v} \in V .
$$

We also define the bilinear forms $b, c$ and $d$ by

$$
\begin{aligned}
b(\mathbf{v}, p) & =-\int_{\Omega} p \operatorname{div} \mathbf{v} \mathrm{dx}, \quad \mathbf{v} \in V, p \in M \\
c(p, \eta) & =\int_{\Omega} p \eta \mathrm{dx}, \quad p, \eta \in M \\
d(\mathbf{u}, \eta) & =\int_{\Omega} T \mathbf{u} \eta \mathrm{dx}, \quad \mathbf{u} \in V, \eta \in M
\end{aligned}
$$

Using these, a weak formulation of (3) is given by: find $[\mathbf{u}, p]$ in $\mathbf{V} \times M$ such that

$$
\begin{aligned}
& a(\mathbf{u}, \mathbf{v})+b(\mathbf{v}, \boldsymbol{p})=\langle\mathbf{f}, \mathbf{v}\rangle, \quad \forall \in \mathbf{V}, \\
& c(p, \eta)+d(\mathbf{u}, \eta)=0, \quad \forall \eta \in M .
\end{aligned}
$$

This will be called the streamline weak formulation.

It is assumed that there is a triangulation $T_{h}=\{K\}$, which is a standard finite element subdivision of $\Omega$ into nonoverlapping triangles $K$ of diameter $h_{K}$. Here, $h=\max _{K} h_{K}$. Let $P(K)$ be the space of linear functions on $K$. Let $\mathbf{V}_{h}$ and $M_{h}$ be defined by

$$
\begin{aligned}
\mathbf{V}_{h} & =\left\{\mathbf{u}_{h} \in \mathbf{V}: \mathbf{u}_{h} \text { is continuous on } \Omega,\left.\mathbf{u}_{h}\right|_{K} \in(P(K))^{2}, \forall K \in T_{h}\right\}, \\
M_{h} & =\left\{p_{h} \in M: p_{h} \text { is continuous on } \Omega,\left.p_{h}\right|_{K} \in P(K), \forall K \in T_{h}\right\} .
\end{aligned}
$$

Let $\Omega_{h}$ be a given polygonal approximation of $\Omega$. Let $\left(\delta_{h}(y), y\right),-1 \leqslant y \leqslant 1$, describe the incoming portion of the boundary $\Gamma_{h}$ of $\Omega_{h}$. Notice that $\delta_{h}(y)$ is a piecewise linear approximation of $\delta(y)=-\sqrt{1-y^{2}}$ for $-1 \leqslant y \leqslant 1$. For $\mathbf{u}_{h} \in \mathbf{V}_{h}$, define

$$
T_{h} \mathbf{u}_{h}(x, y)=\int_{\delta_{h}(y)}^{x} \nabla \cdot \mathbf{u}_{h}(s, y) d s .
$$

Hence $T_{h} \mathbf{u}_{h} \in M_{h}$. We define a difference between $\Gamma_{\text {in }}$ and $\Gamma_{h, \text { in }}$, the incoming portion of $\Gamma_{h}$ :

$$
E_{\Gamma_{\text {in }}}(y)=\left|\delta_{h}(y)-\delta(y)\right|\left(1-y^{2}\right)^{1 / 4}, \quad-1 \leqslant y \leqslant 1 .
$$

If $f$ is a function of only $y$, we denote its $L^{2}$ norm by $\|f\|_{L^{2}[-1,1]} \equiv\left\{\int_{-1}^{1}|f(y)|^{2} d y\right\}^{1 / 2}$. 
Lemma 2.1. Let $q>2$. Then $\left\|\left(T-T_{h}\right) \mathbf{u}\right\|_{0} \leqslant \sqrt{2}\|\nabla \mathbf{u}\|_{1, q}\left\|E_{\Gamma_{\mathrm{in}}}\right\|_{L^{2}[-1,1]}$.

PRoof: Using the Sobolev inequality $H^{1, q}(\Omega) \subset L^{\infty}$,

$$
\begin{aligned}
\left|\left(T-T_{h}\right) \mathbf{u}\right|^{2} & =\left|\int_{\delta(y)}^{\delta_{h}(y)} \nabla \cdot \mathbf{u}(s, y) d s\right|^{2} \\
& \leqslant\|\nabla \mathbf{u}\|_{1, q}^{2}\left|\delta_{h}(y)-\delta(y)\right|^{2} .
\end{aligned}
$$

Hence the result follows easily.

Let $\left\{\left(x_{i}, y_{i}\right): x_{i}^{2}+y_{i}^{2}=1,0 \leqslant i \leqslant n+1\right\}$ be the set of the nodal points of the incoming portion $\Gamma_{h, \text { in }}$ for a given polygonal approximation $\Omega_{h}$ of $\Omega$, where $x_{0}=0$, $y_{0}=-1$, and $x_{n+1}=0, y_{n+1}=1$.

LEMMA 2.2. Let $\bar{x}=\max \left\{\left|x_{1}\right|,\left|x_{n}\right|\right\}$. Then $\left\|E_{\Gamma_{\mathrm{in}}}\right\|_{L^{2}[-1,1]} \leqslant 2 \sqrt{2} \bar{x}^{2+1 / 2}+C h$, where $C$ is independent of $h$.

Proof: We have that

$$
\int_{-1}^{1} E_{\Gamma_{\mathrm{in}}}(y)^{2} d y=\int_{y_{0}}^{y_{1}} E_{\Gamma_{\mathrm{in}}}(y)^{2} d y+\int_{y_{n}}^{y_{n+1}} E_{\Gamma_{\mathrm{in}}}(y)^{2} d y+\sum_{i=2}^{n} \int_{y_{i-1}}^{y_{i}} E_{\Gamma_{\mathrm{in}}}(y)^{2} d y
$$

The second term of the right hand side of the above equation is bounded by

$$
\begin{aligned}
\int_{y_{n}}^{y_{n+1}} E_{\Gamma_{\mathrm{in}}}(y)^{2} d y & \leqslant 4 \int_{y_{n}}^{1}|\delta(y)|^{3} d y \\
& \leqslant 4\left|\delta\left(y_{n}\right)\right|^{3}\left(1-y_{n}\right) \\
& \leqslant 4\left|\delta\left(y_{n}\right)\right|^{5}=4\left|x_{n}\right|^{5}
\end{aligned}
$$

where we used $1-y_{n} \leqslant 1-y_{n}^{2}=x_{n}^{2}$ in the last inequality. Similiarly, the first term of the right hand side of the above equation is bounded by $4\left|x_{1}\right|^{5}$. In order to compute the third term of the above equation, we have, using Lagrange's interpolation formula, $\left|\delta(y)-\delta_{h}(y)\right|=(1 / 2)\left(y_{i}-y\right)\left(y-y_{i-1}\right)\left(1-\xi_{y}^{2}\right)^{-3 / 2}, y_{i-1} \leqslant \xi_{y} \leqslant y_{i},(i=2,3, \ldots, n)$. So

$$
\begin{aligned}
\sum_{i=2}^{n} \int_{y_{i-1}}^{y_{i}} E_{\Gamma_{\text {in }}}(y)^{2} d y & =\frac{1}{4} \sum_{i=2}^{n} \int_{y_{i-1}}^{y_{i}}\left(y-y_{i-1}\right)^{2}\left(y-y_{i}\right)^{2} \frac{1}{\left(1-\xi_{y}^{2}\right)^{3}} \sqrt{1-y^{2}} d y \\
& \leqslant C h^{2} \sum_{i=2}^{n} \int_{y_{i-1}}^{y_{i}}\left(1-y^{2}\right)^{-1 / 2} d y \\
& \leqslant C h^{2}
\end{aligned}
$$

where $\mathrm{C}$ is independent of $h$. This finishes our proof. 
The discrete bilinear form of " $d$ " is defined on $\mathrm{V}_{h} \times M_{h}$ in the following way: $d_{h}\left(\mathbf{u}_{h}, \eta\right)=\left\langle T_{h} \mathbf{u}_{h}, \eta\right\rangle, \eta \in M_{h}$. Then the finite element method corresponding to (4) is given by: find $\left[\mathbf{u}_{h}, p_{h}\right] \in \mathrm{V}_{h} \times M_{h}$ such that

$$
\begin{aligned}
a\left(\mathbf{u}_{h}, \mathbf{v}\right)+b\left(\mathbf{v}, p_{h}\right) & =\langle\mathbf{f}, \mathbf{v}\rangle, & & \forall \mathbf{v} \in \mathbf{V}_{h}, \\
c\left(p_{h}, \eta\right)+d_{h}\left(\mathbf{u}_{h}, \eta\right) & =0, & & \forall \eta \in M_{h} .
\end{aligned}
$$

Since the definition of $T_{h}$ includes an integration, the finite element matrix coming from (6) is not sparse. It also is easily proven that from $(6)_{b}, p_{h}(x, y)+T_{h} \mathbf{u}_{h}(x, y)=0$ on $\Omega$.

LEMMA 2.3. If $\mu$ is sufficiently large, then there is a unique solution $\left[\mathbf{u}_{h}, p_{h}\right] \in$ $\mathbf{V}_{h} \times M_{h}$ of (6). Also, there is a constant $C$, not depending on $h$, such that if $\left[\mathbf{u}_{h}, p_{h}\right]$ satisfies (6), then

$$
\left\|\mathbf{u}_{h}\right\|_{1}+\left\|p_{h}\right\|_{0} \leqslant C\|\mathbf{f}\|_{0}
$$

PROOF: Let $\eta=p_{h} \in M_{h}$ in $(6)_{b}$. So we have $c\left(p_{h}, p_{h}\right)+d_{h}\left(\mathbf{u}_{h}, p_{h}\right)=0$ and easily get $\left\|p_{h}\right\|_{0} \leqslant\left\|\nabla \mathbf{u}_{h}\right\|_{0}$. Next, let $\mathbf{v}=\mathbf{u}_{h} \in \mathbf{V}_{h}$ in $(6)_{a}$. Then $a\left(\mathbf{u}_{h}, \mathbf{u}_{h}\right)+b\left(\mathbf{u}_{h}, p_{h}\right)=$ $\left\langle\mathbf{f}, \mathbf{u}_{h}\right\rangle$ and we get easily $\mu_{0}\left\|\nabla \mathbf{u}_{h}\right\|_{0}^{2} \leqslant C\left\|\nabla \mathbf{u}_{h}\right\|_{0}\left\|\mathbf{u}_{h}\right\|_{0}+\left\|p_{h}\right\|_{0}\left\|\nabla \mathbf{u}_{h}\right\|_{0}+\|\mathbf{f}\|_{0}\left\|\mathbf{u}_{h}\right\|_{0}$, where $C=C\left(|\mathbf{U}|_{\infty}\right), \mu_{0}=\min \{\mu, \mu+\nu\}$. Finally, choosing a Poincaré constant $\vec{C}$ such that $\left\|\mathbf{u}_{h}\right\|_{0} \leqslant \bar{C}\left\|\nabla \mathbf{u}_{h}\right\|_{0}, \mathbf{u}_{h} \in \mathbf{V}_{h}$, and assuming that $\mu$ is large enough, we have $\left\|\nabla \mathbf{u}_{h}\right\|_{0} \leqslant C\|\mathbf{f}\|_{0}, C=C\left(|\mathbf{U}|_{\infty}, \bar{C}, \mu_{0}\right)$. Thus, we obtain (7). A similiar argument shows the existence and uniqueness of the solution of $(6)$.

Let $\widehat{\mathbf{u}} \in V_{h}$ be an approximation to $\mathbf{u}$, and let

$$
\widehat{p}(x, y)=-T_{h} \widehat{\mathbf{u}}(x, y) .
$$

REMARK. One need a requirement on the triangulation "no triangle has a horizontal side" in establishing that $\widehat{p} \in M_{h}$. However as will be seen this requirement is not necessary to obtain an error estimate for our finite element method. With this requirement, one easily see that the function $\hat{p}$ belongs to $M_{h}$.

We now give an error analysis of the "streamline" finite element method. Let $[\mathbf{u}, p]$ be the true solution of $(4)$ and let $\left[\mathbf{u}_{h}, p_{h}\right]$ be the finite element solution of $(6)$.

TheOREM 2.4. Let $q>2$. If $\mu$ is sufficiently large, then there is a constant $K$ depending on $\mu, \nu$, and $\mathbf{U}$ such that

$$
\left\|\mathbf{u}-\mathbf{u}_{h}\right\|_{1}+\left\|p-p_{h}\right\|_{0} \leqslant K\left\{\inf \left[\|\nabla(\mathbf{u}-\widehat{\mathbf{u}})\|_{0}\right]+\|\nabla \mathbf{u}\|_{1, q}\left\|E_{\Gamma_{\mathrm{in}}}\right\|_{L^{2}[-1,1]}\right\}
$$

where the infimum is taken over all $\widehat{\mathbf{u}} \in \mathbf{V}_{h}$.

Proof: Suppose first that the triangulation is such that no triangle has a horizontal side. Let $\widehat{\mathbf{u}} \in \mathbf{V}_{h}$, and let $\widehat{p}=-T_{h} \widehat{\mathbf{u}}$. By the assumption on the triangulation, 
$\widehat{p} \in M_{h}$. Using (4) and (6), we have $a\left(\mathbf{u}-\mathbf{u}_{h}, \mathbf{v}\right)+b\left(\mathbf{v}, p-p_{h}\right)=0, \quad \forall \mathbf{v} \in \mathbf{V}_{h}$ and $c\left(p-p_{h}, \eta\right)+d(\mathbf{u}, \eta)-d_{h}\left(\mathbf{u}_{h}, \eta\right)=0, \quad \forall \eta \in M_{h}$. Hence one has

$$
\begin{aligned}
a\left(\mathbf{u}_{h}-\widehat{\mathbf{u}}, \mathbf{v}\right)+b\left(\mathbf{v}, p_{h}-\widehat{p}\right) & =a(\mathbf{u}-\widehat{\mathbf{u}}, \mathbf{v})+b(\mathbf{v}, p-\widehat{p}), & & \forall \mathbf{v} \in \mathbf{V}_{h} \\
c\left(p_{h}-\widehat{p}, \eta\right)+d_{h}\left(\mathbf{u}_{h}-\widehat{\mathbf{u}}, \eta\right) & =c(p-\widehat{p}, \eta)+d(\mathbf{u}, \eta)-d_{h}(\widehat{\mathbf{u}}, \eta), & & \forall \eta \in M_{h}
\end{aligned}
$$

Picking $\eta=p_{h}-\widehat{p} \in M_{h}$ in $(10)_{b}$ and taking $d_{h}\left(\mathbf{u}_{h}-\widehat{\mathbf{u}}, p_{h}-\widehat{p}\right)$ to the right hand side,

$$
\begin{aligned}
\left\|p_{h}-\widehat{p}\right\|_{0} & \leqslant\|p-\widehat{p}\|_{0}+\left\|T \mathbf{u}-T_{h} \widehat{\mathbf{u}}\right\|_{0}+\left\|T_{h} \mathbf{u}_{h}-T_{h} \widehat{\mathbf{u}}\right\|_{0} \\
& \leqslant C_{1}\left(\left\|\nabla\left(\mathbf{u}_{h}-\widehat{\mathbf{u}}\right)\right\|_{0}+\|\nabla(\mathbf{u}-\widehat{\mathbf{u}})\|_{0}+\|p-\widehat{p}\|_{0}\right) \\
& +C_{1}\left\|\left(T-T_{h}\right) \mathbf{u}\right\|_{0}
\end{aligned}
$$

The constant $C_{1}$ depends on $\left\|T_{h}\right\|$. Since the polygonal domain is contained in $\Omega$, one see that $\left\|T_{h}\right\| \leqslant\|T\|$. Since $\|T\|$ depends only on $\Omega$, one concludes that the constant $C_{1}$ in (11) depends only on $\Omega$. Next pick $\mathbf{v}=\mathbf{u}_{h}-\widehat{\mathbf{u}} \in \mathbf{V}_{h}$ in $(10)_{a}$. So

$$
\begin{aligned}
a\left(\mathbf{u}-\widehat{\mathbf{u}}, \mathbf{u}_{h}-\widehat{\mathbf{u}}\right)+b\left(\mathbf{u}_{h}-\widehat{\mathbf{u}}, p-\widehat{p}\right) \leqslant & (\mu+|\nu|)\|\nabla(\mathbf{u}-\widehat{\mathbf{u}})\|_{0}\left\|\nabla\left(\mathbf{u}_{h}-\widehat{\mathbf{u}}\right)\right\|_{0} \\
& +C\|\nabla(\mathbf{u}-\widehat{\mathbf{u}})\|_{0}\left\|\mathbf{u}_{h}-\widehat{\mathbf{u}}\right\|_{0} \\
& +\|p-\widehat{p}\|_{0}\left\|\nabla\left(\mathbf{u}_{h}-\widehat{\mathbf{u}}\right)\right\|_{0}
\end{aligned}
$$

where $C=C\left(|\nabla \mathbf{U}|_{\infty}\right)$. Choose a Poincaré constant $\bar{C}$ such that $\bar{C}\left\|\mathbf{u}_{h}-\widehat{\mathbf{u}}\right\|_{0} \leqslant$ $\left\|\nabla\left(\mathbf{u}_{h}-\widehat{\mathbf{u}}\right)\right\|_{0}$. Thus, using (12),

(13) $a\left(\mathbf{u}-\widehat{\mathbf{u}}, \mathbf{u}_{h}-\widehat{\mathbf{u}}\right)+b\left(\mathbf{u}_{h}-\widehat{\mathbf{u}}, p-\widehat{p}\right) \leqslant C\left(\|\nabla(\mathbf{u}-\widehat{\mathbf{u}})\|_{0}+\|p-\widehat{p}\|_{0}\right)\left\|\nabla\left(\mathbf{u}_{h}-\widehat{\mathbf{u}}\right)\right\|_{0}$, where $C=C\left(\mu, \nu, \bar{C},\|\mathbf{U}\|_{1, \infty}\right)$. Furthermore we have

$$
\begin{aligned}
a\left(\mathbf{u}_{h}-\widehat{\mathbf{u}}, \mathbf{u}_{h}-\widehat{\mathbf{u}}\right)+b\left(\mathbf{u}_{h}-\widehat{\mathbf{u}}, p_{h}-\widehat{p}\right) \geqslant & \mu_{0}\left\|\nabla\left(\mathbf{u}_{h}-\widehat{\mathbf{u}}\right)\right\|_{0}^{2}-\frac{1}{2}|\operatorname{div} \mathbf{U}|_{\infty}\left\|\mathbf{u}_{h}-\widehat{\mathbf{u}}\right\|_{0}^{2} \\
& -\left\|p_{h}-\widehat{p}\right\|_{0}\left\|\nabla\left(\mathbf{u}_{h}-\widehat{\mathbf{u}}\right)\right\|_{0}
\end{aligned}
$$

where $\mu_{0}=\min \{\mu, \mu+\nu\}$. Next, using (11), we have

$$
\begin{aligned}
a\left(\mathbf{u}_{h}-\widehat{\mathbf{u}}, \mathbf{u}_{h}-\widehat{\mathbf{u}}\right)+b\left(\mathbf{u}_{h}-\widehat{\mathbf{u}}, p_{h}-\widehat{p}\right) \geqslant\left(M_{0}-C_{1}\right)\left\|\nabla\left(\mathbf{u}_{h}-\widehat{\mathbf{u}}\right)\right\|_{0}^{2} \\
-C_{1}\left\|\nabla\left(\mathbf{u}_{h}-\widehat{\mathbf{u}}\right)\right\|_{0}\left(\|\nabla(\mathbf{u}-\widehat{\mathbf{u}})\|_{0}+\|p-\widehat{p}\|_{0}+\left\|\left(T-T_{h}\right) \mathbf{u}\right\|_{0}\right),
\end{aligned}
$$

where $M_{0}=\mu_{0}-(1 / 2) \bar{C}^{-1}|\operatorname{div} \mathbf{U}|_{\infty}$ and $C_{1}$ is the constant in (11). Combining (11), (13) and (15), we get

$$
\left(M_{0}-C_{1}\right)\left\|\nabla\left(\mathbf{u}_{h}-\widehat{\mathbf{u}}\right)\right\|_{0} \leqslant C\left(\|\nabla(\mathbf{u}-\widehat{\mathbf{u}})\|_{0}+\|p-\hat{p}\|_{0}+\left\|\left(T-T_{h}\right) \mathbf{u}\right\|_{0}\right),
$$


where $C=C\left(\Omega, \mu, \nu, \bar{C},\|\mathbf{U}\|_{1, \infty}\right)$. The constant $M_{0}-C_{1}$ is positive for a sufficiently large $\mu$. Finally using (11) and (16), we obtain

$$
\left\|\nabla\left(\mathbf{u}_{h}-\widehat{\mathbf{u}}\right)\right\|_{0}+\left\|p_{h}-\widehat{p}\right\|_{0} \leqslant C_{2}\left(\|\nabla(\mathbf{u}-\widehat{\mathbf{u}})\|_{0}+\|p-\widehat{p}\|_{0}+\left\|\left(T-T_{h}\right) \mathbf{u}\right\|_{0}\right),
$$

where $C_{2}=C\left(\Omega, \mu, \nu, \bar{C},\|\mathbf{U}\|_{1, \infty}, M_{0}\right)$. Thus, using the triangle inequality, we have

$$
\left\|\nabla\left(\mathbf{u}-\mathbf{u}_{h}\right)\right\|_{0}+\left\|p-p_{h}\right\|_{0} \leqslant C_{2}\left(\|\nabla(\mathbf{u}-\widehat{\mathbf{u}})\|_{0}+\|p-\widehat{p}\|_{0}+\left\|\left(T-T_{h}\right) \mathbf{u}\right\|_{0}\right)
$$

Since $\|p-\widehat{p}\|_{0} \leqslant C\left(\|\nabla(\mathbf{u}-\widehat{\mathbf{u}})\|_{0}+\left\|\left(T-T_{h}\right) \mathbf{u}\right\|_{0}\right)$, we have

$$
\left\|\mathbf{u}-\mathbf{u}_{h}\right\|_{1}+\left\|p-p_{h}\right\|_{0} \leqslant C_{2}\left(\|\nabla(\mathbf{u}-\widehat{\mathbf{u}})\|_{0}+\left\|\left(T-T_{h}\right) \mathbf{u}\right\|_{0}\right) .
$$

Therefore we conclude that

$$
\left\|\mathbf{u}-\mathbf{u}_{h}\right\|_{1}+\left\|p-p_{h}\right\|_{0} \leqslant C_{2}\left\{\inf \left[\|\nabla(\mathbf{u}-\widehat{\mathbf{u}})\|_{0}\right]+\left\|\left(T-T_{h}\right) \mathbf{u}\right\|_{0}\right\},
$$

where $C_{2}=C\left(\Omega, \mu, \nu, \bar{C},\|\mathbf{U}\|_{1, \infty}, M_{0}\right)$, and the infimum is taken over all $\hat{\mathbf{u}} \in \mathbf{V}_{h}$. Thus, using Lemma 2.1, we can finish our proof in the case that no triangle in the triangulation has a horizontal side. Since the constant $K$ in (9) has been shown to be independent of the triangulation, the result holds for any triangulation by a limiting argument.

We next use the following approximation property to give a corollary to the theorem.

$$
\inf _{\mathbf{v} \in \mathbf{V}_{h}}\|\mathbf{u}-\mathbf{v}\|_{1} \leqslant C h^{k}\|\mathbf{u}\|_{k+1}, \quad \forall \mathbf{u} \in \mathbf{V} \cap\left(H^{k+1}(\Omega)\right)^{2}
$$

where $C$ is a constant independent of $h$.

Corollary 2.5. Let $q>2$. Assume that the conditions in Theorem 2.4 hold. Then there exists a constant $K$, depending on $\mu, \nu$ and $\mathrm{U}$ such that the finite element solution $\left[\mathbf{u}_{h}, p_{h}\right]$ of $(6)$ satisfies

$$
\left\|\mathbf{u}-\mathbf{u}_{h}\right\|_{1}+\left\|p-p_{h}\right\|_{0} \leqslant K h\|\mathbf{u}\|_{2, q}
$$

PRoOF: Theorem 2.4 gives $\left\|\mathbf{u}-\mathbf{u}_{h}\right\|_{1}+\left\|p-p_{h}\right\|_{0} \leqslant K\left(h+\left\|E_{\Gamma_{\mathrm{in}}}\right\|_{L^{2}[-1,1]}\right)\|\mathbf{u}\|_{2, q}$. Hence the inequality (22) follows easily by Lemma 2.2 .

We note that with a large condition on $\mu$, the inequality $\left\|\mathbf{u}-\mathbf{u}_{h}\right\|_{1}+\left\|p-p_{h}\right\|_{0} \leqslant$ $K h$ for a positive constant $K$ can be obtained easily. 


\section{REFERENCES}

[1] R.A. Adams, Sobolev spaces (Academic Press, New York, 1975).

[2] D.N. Arnold, F. Brezzi and M. Fortin, 'A stable finite element for the Stokes equations', Calcolo 21 (1984), 337-344.

[3] R.B. Kellogg and B. Liu, 'A finite element method for the compressible Stokes equations', SIAM J. Numer. Anal. 33 (1996), 780-788.

[4] J.R. Kweon, Compressible Navier-Stokes Equations in two dimensions with inflow boundary condition and Numerical Experiments, Ph.D Thesis (University of Maryland, College Park, 1995).

[5] J.R. Kweon, R.B. Kellogg, 'Compressible Navier-Stokes Equations in a bounded domain with inflow boundary condition', SIAM J. Math. Anal. 28 (1997), 94-108.

Department of Mathematics

College of Natural Science

Pusan Women's University

Pusan 617-736

Korea 